\title{
Erratum zu: Safety und Security
}

\author{
Michael Waidner
}

\section{Erratum zu: Digitalisierung \\ https://doi.org/10.1007/978-3-662-55890-4_16}

Das Kapitel Safety und Security wurde von Prof. Dr. Michael Waidner und Prof. Dr. Claudia Eckert gemeinschaftlich erstellt. Die Autorenschaft des genannten Kapitels wurde um folgenden Namen ergänzt:

Prof. Dr. Claudia Eckert

Fraunhofer-Institut für Angewandte und Integrierte Sicherheit AISEC

Wir bitten, die korrigierte Autorenschaft zu beachten.

Die aktualisierte Originalversion des Kapitels ist verfügbar unter:

https://doi.org/10.1007/978-3-662-55890-4_16

(C) Springer-Verlag GmbH Deutschland 2018

R. Neugebauer (Hrsg.) Digitalisierung, https://doi.org/10.1007/978-3-662-55890-4_23 